\title{
PLANTAE UXPANAPAE I. COLUBRINA JOHNSTONII SP. NOV. (RHAMNACEAE) *
}

\author{
TOM WENDT**
}

\begin{abstract}
RESUMEN
El presente artículo representa el primero de una serie con descripciones de especies nuevas e información relativa a la flora de la Zona de Uxpanapa del sureste de Veracruz y partes colindantes de Oaxaca, México. Se describe la especie nueva Colubrina jobnstonii (Rhamnaceae), relacionada con C. elliptica y otras especies. E1 recuento cromosómico de $n=16$ para la especie nueva es extraordinario para la familia y representa el primero para el género.
\end{abstract}

\section{ABSTRACT}

The present article is the first of a series concerning new species and other information on the plants of the Zona de Uxpanapa, an area of lowland rain forest in the extreme southeast of Veracruz and adjacent parts of Oaxaca, México. Colubrina jobnstonii sp. nov. (Rhamnaceae) is described; it is related to C. elliptica and other species. The chromosome count of $n=16$ for the new species is extraordinary for the family and represents the first count for Colubrina.

La Zona de Uxpanapa comprende un área de cerca de $2,000 \mathrm{~km}^{2}$ situada en la vertiente del Golfo del Istmo de Tehuantepec, en el extremo sureste de Veracruz y partes colindantes de Oaxaca (Fig. 1). Esta zona de tierras rbajas corresponde en general al Distrito de Drenaje de Uxpanapa de la Comisión del Papaloapan, y está delimitada, en términos muy generales, al oeste por el Río Corte ( $R$. Coatzacoalcos), al sur por el Río Verde y la Sierra de Tres Picos (Sierra de Chimalapa), al este por las sierras calizas al este del Río Uxpanapa y, al norte, por líneas arbitrarias de diferentes latitudes en diferentes partes del área. El área esta dividida entre las cuencas de los

* Trabajo aceptado para su publicación en marzo de 1983.

** Centro de Botónica Colegio de Postgraduados 56230 Chapingo, México, México. 
BOLETIN DE LA SOCIEDAD BOTANICA DE MEXICO No. 44, 1983

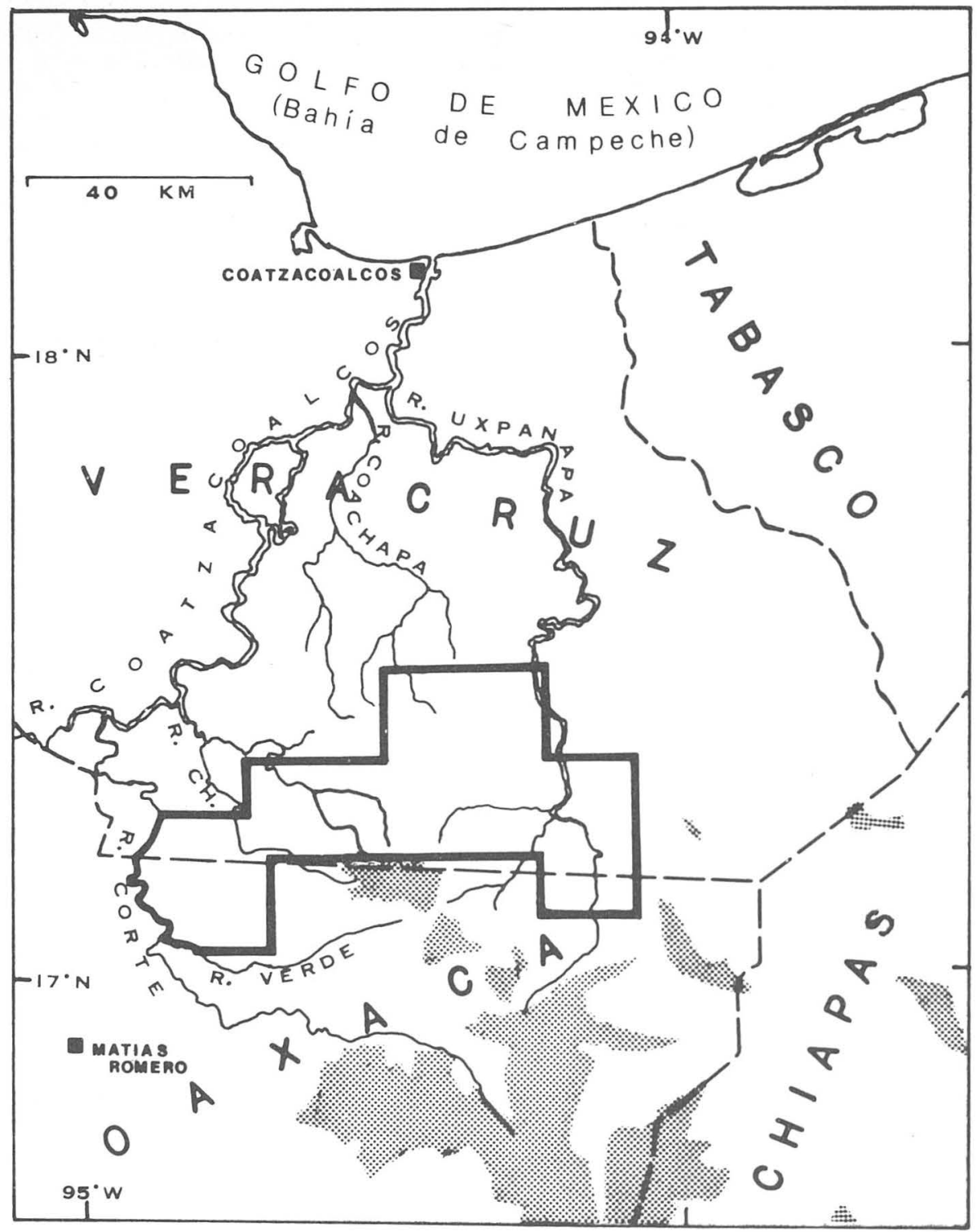

Figura 1. Ubicación de la Zona de Uxpanapa (línea gruesa). Areas sombreadas representan sierras de más de mil metros de elevación. R. Ch. = Río Chalchijapa. Mapa arreglado de varias fuentes. 
Ríos Corte, Chalchijapa, Coachapa, y Uxpanapa, los cuales son afluentes del Río Coatzacoalcos. Se encuentran en la región substratos geológicos diversos, incluyendo zonas de caliza. cárstica en el norte, la parte baja de la vertiente norte de la sierra granítica al sur, y sierras y lomas calizas, con planicies aluviales en el centro. La región presenta una precipitación total anual elevada, con ca. $2850 \mathrm{~mm}$ en el oeste aumentando rápidamente hacia el este, con ca. $4000 \mathrm{~mm}$ en el centro y ca. $4400 \mathrm{~mm}$ en el este (Comisión del Papaloapan, datos inéditos) y seguramente más en las sierras, con una época de sequía bien marcada en la primavera. La vegetación primaria de la zona comprende selvas tropicales de diversos tipos, correspondiendo en su mayoría aproximadamente a las selvas alta perennifolia y alta-mediana subperennifolia de Miranda y Hernández X. (1963).

Márquez R. et al. (1981) han publicado una lista florística y una clasificación de los tipos de vegetación de una parte de la Zona de Uxpanapa, pero la composición florística de las selvas del área queda muy imperfectamente conocida. Debido a que esta zona y las áreas colindantes presentan en la actualidad una de las extensiones más grandes de selva tropical virgen y semi-virgen en México, el Centro de Botánica del Colegio de Postgraduados inició en 1980 un proyecto para estudiar la flora leñosa de la región. Las colectas de 1980 al presente han rendido un gran número de especies nuevas para la ciencia, indicando que la región representa un centro de endemismo para las selvas tropicales del país. Algunas de estas especies serán publicadas por especialistas de varios grupos, y el resto en una serie de artículos bajo el nombre, "Plantae Uxpanapae", de los cuales el presente es el primero.

Colubrina (secc. Cowania) jobnstonii Wendt, sp. nov., ex affinitate C. ellipticae (Sw.) Brizicky \& Stern et $C$. beteroneuris (Griseb.) Standley, ab utroque laminis (2.8-) 3-4(-5.5)-plo (nec 1.5-2.7(-3.3)-plo) longioribus quam latioribus, stipulis persistentibus, sepalis erectis (nec patentibus nec reflexis), fructibus grandioribus 6.5-8 (nec 5-7) $\mathrm{mm}$ longis, et praeterea a priore laminis anguste obovatis vel oblanceolatis (nec ovatis nec ovato-ellipticis), a posteriore absentia spinarum recedit (Fig. 2).

Arbusto o arbolito de $1-4 \mathrm{~m}$ de alto; ramas jóvenes pubescentes con pelos encorvado-adpresos, rojo-pardos, pronto glabrescentes, usualmente finamente estriadas y con pequeñas verrugas lenticelares pustuliformes; lámina obovada u obovadalanceolada (raras veces elíptica en hojas pequeñas), $4-15 \mathrm{~cm}$ de largo, $1.3-4 \mathrm{~cm}$ de ancho, (2.8-) 3-4 (-5.5) veces más larga que ancha, la base cuneada o aguda, distalmente acuminada a un ápice redondeado o emarginado y frecuentemente mucronulado, margen entero con una glándula elíptica o lineal marginal a $0.5-4 \mathrm{~mm}$ de la base a cada lado, sin otras glándulas, superficies discoloras, haz verde, glabro, con una vena central y a veces algunas venas secundarias pubescentes o finalmente glabras, envés pálido, ligeramente pubescente especialmente en las venas, vena central fuertemente acanalada en la base adaxialmente, venas secundarias camptódromas, 4-9 a cada lado, espaciadas regularmente o distalmente más apretadas; peciolo 4-17 mm de largo, pubescente o glabrescente, acanalado adaxialmente; estipulas $2.5-5.5 \mathrm{~mm}$ de largo, subuladas con la vena central pubescente, engrosada y subespinescente, al principio verde pálidas tornándose rápidamente rojo-pardo obscuras y endureciéndose, persistentes, margen finamente eroso-ciliolado por lo menos en la base. Flores en fascículos axilares en nudos foliosos o desnudos, cada fascículo formado por una inflorescencia ramificada en la base con pocos a varios ejes reducidos, indeterminados, bracteados, hasta $5 \mathrm{~mm}$ de largo, cada uno con varias flores, fascículo total con pocas 


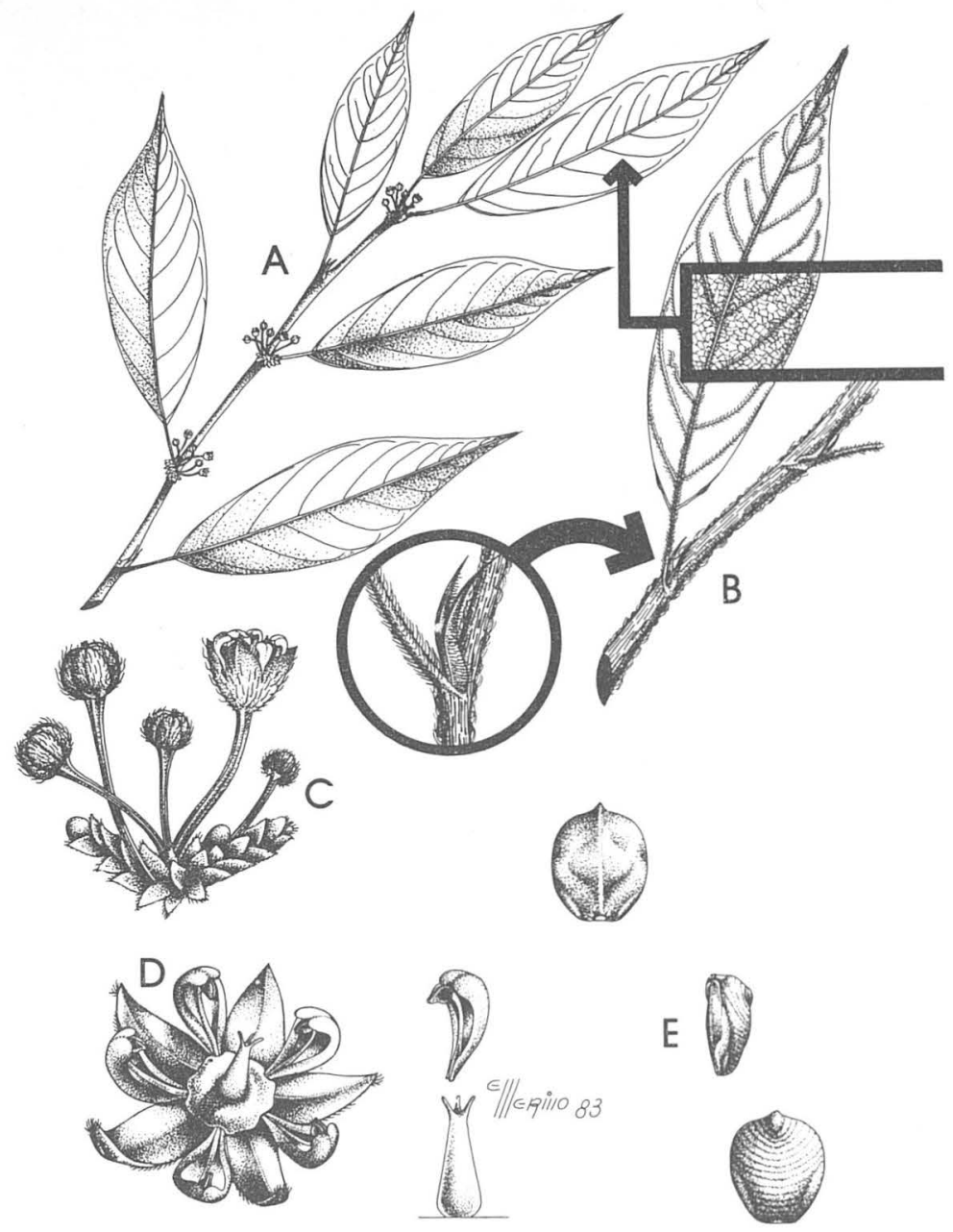

Figura 2. Colubrina jobnstonii Wendt A. Ramita con inflorescencias; hojas sombreadas muestran el haz (X 0.45). B. Detalles de ramita y envés de la hoja (X 1), con detalles de estípulas (X 3). C. Parte de una inflorescencia, con una flor un poco antes de la antesis (X 4.5). D. Flor, con los sépalos más reflexos que lo normal para mostrar los detalles ( $\mathrm{X}$ 8), con pistilo y un pétalo y su estambre aislados a la derecha. E. Semilla, en vista ventral (arriba), lateral, y dorsal (X 2.2). (A-D, Wendt et. al. 4039; E, Wendt et. al. 3678). Ilustración por Eduardo Merino. 
a muchas (usualmente 10-20) flores, frecuentemente apareciendo excéntrico en la axila; brácteas anchamente ovadas, oblicuas, cilioladas, hasta ca. $1 \mathrm{~mm}$ de largo; botones anchamente truncado-turbinados a transversalmente elipsoides, cada sépalo del botón marcadamente sulcado, por lo tanto el botón 5 -sulcado con 5 líneas sulcadas menores entre los sépalos valvados adyacentes; pedicelos $3-8 \mathrm{~mm}$ de largo, ligeramente puberulentos o glabrescentes, alargándose en el fruto hasta 10-21 mm; bipanto 2-3 $\mathrm{mm}$ de ancho, ligeramente pubescente con pelos encorvado-adpresos; sépalos erectos, deltoides, ca. $1.5 \mathrm{~mm}$ de largo, fuertemente engrosados adaxialmente en el centro, abaxialmente planos o algo sulcados con pelos esparcidos antrorsos y un mechón apical de pelos cortos; pétalos blancos, $1-1.5 \mathrm{~mm}$ de largo, ca. $1 \mathrm{~mm}$ de ancho pero marcadamente cimbiformes y así $0.5 \mathrm{~mm}$ de ancho in vivo, en la base abruptamente angostados en una uña pequeña, ápice truncado-emarginado, cada pétalo envolviendo un estambre pero pronto caedizo; estambres un poco más largos que los pétalos; disco grande; estilo dividido en 3 lóbulos cónicos 1/4 o menos de su longitud. Fruto 6.5-8 $\mathrm{mm}$ de largo, 8-9 mm de grueso, oblato-obovoide, ligeramente 3- (raras veces 4-) lobulado, con hipanto $1 / 4-1 / 3$ de la longitud del fruto, pronto dehiscente dejando una columela pequeña irregular con 3 alas; semilla 1 por lóculo, 4.3-6 mm de largo, 3.5-5 mm de ancho, 2.3-2.8 $\mathrm{mm}$ de grosor (diámetro dorsiventral) en el ápice, menos en la base, obovoide y algo comprimida dorsiventralmente, cara ventral algo a fuertemente diedra con cresta longitudinal central la cual continúa radialmente en el ápice, cara dorsal fuertemente convexa, corcovada, en la base algo muescada o redonda, gris-pardo obscura o rojo-parda, a veces moteada; endospermo delgado, cotiledones gruesos, verdes; número cromosómico $n=16$ (Fig. 2, 3).

TIPO: Veracruz: Municipio de Minatitlán. Zona Uxpanapa: $13.7 \mathrm{~km}$ al E de La Laguna sobre la terracería a Uxpanapa, después $7.2 \mathrm{~km}$ al $\mathrm{N}$ sobre el camino no completo (brecha 93) a Belisario Domínguez, en una depresión cárstica donde emerge y sumerge un río; área cárstica con selva alta-mediana, $17^{\circ} 20^{\prime} \mathrm{N}, 94^{\circ} 22^{\prime} \mathrm{O}, 120 \mathrm{msnm}$, 26 sept. 1980, Wendt, Villalobos, Lott, Lara, Muñoz y Navarrete 2756 (holotipo, MEXU; isotipos: BM, CAS, CHAPA, ENCB, F, MO, NY, TEX, XAL).

PARATIPOS: Veracruz: Mpio. Minatirlán, Zona Uxpanapa: Localidad tipo, 12 julio 1980, Wendt, Villalobos y Navarrete 2535 (CHAPA, ENCB, MEXU, TEX, XAL); Localidad tipo, Wendt, Villalobos y Olmstead 2846 (CHAPA, ENCB, MEXU, TEX, $\mathrm{XAL})$; Río Uxpanapa, desde el poblado de Uxpanapa río arriba hasta los límites con Oaxaca, $17^{\circ} 12^{\prime} \mathrm{N}, 94^{\circ} 10^{\prime} \mathrm{O}, 130 \mathrm{msnm}$, 14 julio 1980, Wendt, Villalobos y Lara 2576-A (CHAPA); $2 \mathrm{~km}$ al $\mathrm{N}$ de Uxpanapa (Poblado 12 ) sobre el camino al Poblado 13, en arroyo seco, $17^{\circ} 14^{\prime} \mathrm{N}, 94^{\circ} 13^{\prime} \mathrm{O}, 130 \mathrm{msnm}, 14$ marzo 1982 , Wendt, Villalobos y Navarrete 3678 (CAS, CHAPA, ENCB, F, MEXU, MO, NY, TEX, $\mathrm{U}, \mathrm{XAL}$ ); Arroyo Naranjal, $8 \mathrm{~km}$ al E del Río Grande sobre la terracería La LagunaUxpanapa, $17^{\circ} 15^{\prime} 30^{\prime \prime} \mathrm{N}, 9^{\circ} 19^{\prime} \mathrm{O}, 140 \mathrm{msnm}, 15$ marzo 1982, Wendt, Villalobos y Navarrete 3702 (BM, CSAT, CHAPA, ENCB, GH, INIF, MEXU, TEX, US, UTD, $\mathrm{XAL}) ; 13.7 \mathrm{~km}$ al E de La Laguna, terracería a Uxpanapa, después $6.5-7 \mathrm{~km}$ al $\mathrm{N}$ por brecha $93,17^{\circ} 20^{\prime} \mathrm{N}, 94^{\circ} 23^{\prime} \mathrm{O}, 130 \mathrm{msnm}, 30$ sept. 1982, Wendt, Villalobos y Navarrete 4039 (CAS, CSAT, CHAPA, ENCB, MEXU, TEX, XAL).

Hábitat: Zonas cársticas y calizas con precipitación promedio anual de más de 4,000 $\mathrm{mm}$, en selva alta perennifolia, selva alta-mediana subperennifolia, o selva riparia, usualmente en las orillas de arroyos o ríos, frecuentemente entre piedras.

Máxima de floración: julio-noviembre. Máxima de fructificación: febrero-marzo. 
BOLETIN DE LA SOCIEDAD BOTANICA DE MEXICO No. 44, 1983
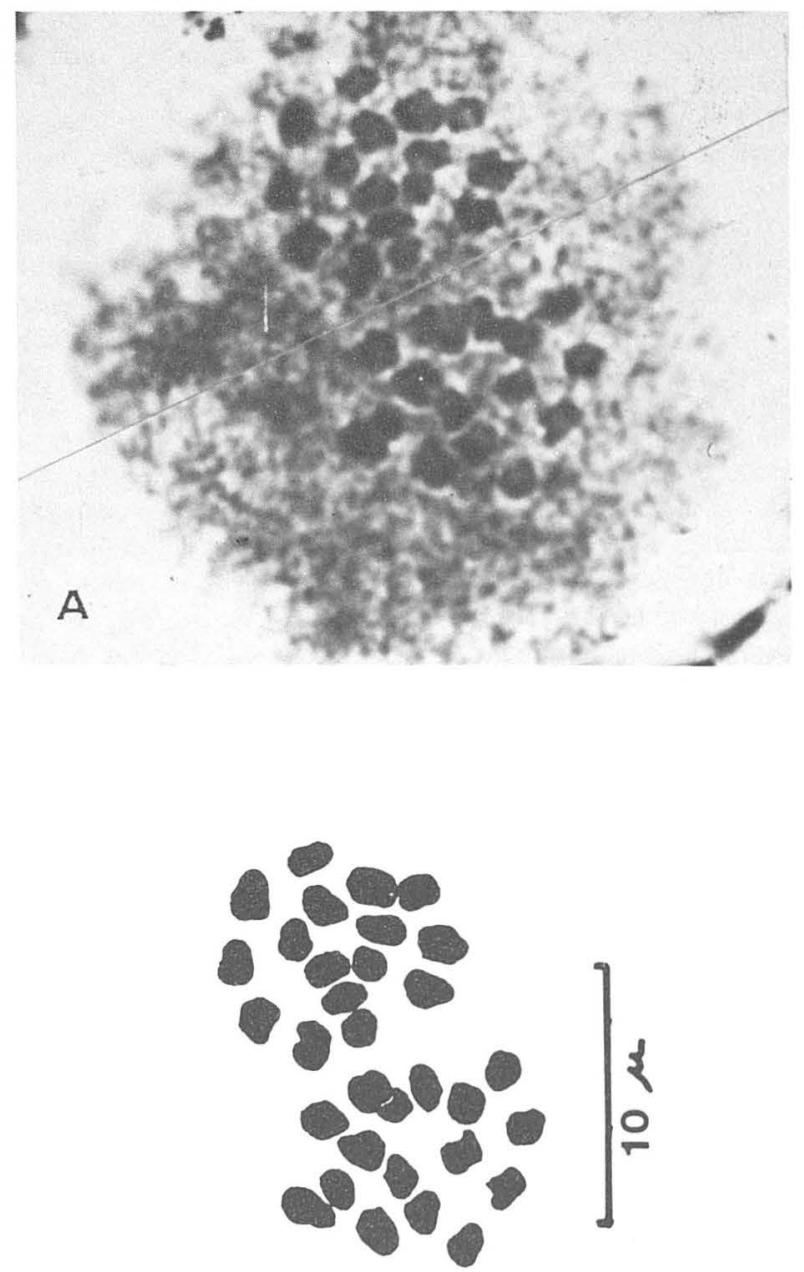

B

Figura 3. Cromosomas meióticos de célula madre del polen de Colubrina jobnstonii, metafase II, $n=16$. A. Microfotografía. B. Dibujo con cámara clara, (Wendt et. al. 4039). 
Siguiendo la clasificación infragenérica de la monografía de Colubrina de M.C. Johnston (1971), C. jobnstonii pertenece a la sección Cowania M.C. Johnst., por las hojas enteras con glándulas marginales. Las especies más emparentadas probablemente son C. elliptica (Sw.) Brizicky \& Stern, C. beteronuera (Griseb.) Standley, y tal vez C. spinosa Donn.-Sm., con relaciones más estrechas con la primera. La especie nueva es distinta de todas éstas por sus estípulas persistentes, las hojas relativamente más angostas, y los sépalos erectos en la antesis; además, se diferencia de cada una de las especies antes mencionadas por otras características (Cuadro 1). Ecológicamente, es muy distinta de $C$. elliptica y $C$. heteroneura, las dos especies que habitan en la misma parte de México; estas últimas especies se encuentran en matorrales y selvas secas muy disímiles a la selva tropical lluviosa en la cual se encuentra $C$. jobnstonii. C. spinosa var. spinosa parece ser algo similar a $C$. jobnstonii en este respecto, encontrándose en las tierras bajas húmedas de Nicaragua, Costa Rica, y Panamá (Johnston, 1971).

La clave parcial para la secc. Cowania que se presenta a continuación incluye modificaciones a la de Johnston (1971). La primera opción de la dicotomía se ha cambiado para acomodar no sólo la condición de solamente un par de glándulas marginales presentes en $C$. jobnstonii, sino también la misma condición presente casi siempre en $C$. beteroneura y frecuentemente en $C$. elliptica.

\section{CLAVE PARCIAL DE COLUBRINA SECT. COW ANIA (modificado de Johnston, 1971)}

Glándulas del margen de la hoja, cuando presentes, más de 2, o cuando 2, a una distancia de $0.5 \mathrm{~mm}$ o más de la base de la vena central (en la mayoría de las hojas) e inconspicuas, anulares a lineares.

Hojas pequeñas, láminas $4-15(-20) \mathrm{mm}$ de ancho ..... (C. verrucosa, C. viridis)

Hojas más grandes, (13-) 20-60 mm de ancho.

Hojas 3-4 o más veces más largas que anchas; estípulas persistentes; sépalos erectos en antesis; frutos $6.5-8 \mathrm{~mm}$ de largo ........... C. jobnstonii Hojas 1.5-2.7(-3.3) veces más largas que anchas; estípulas caedizas; sépalos patentes o reflexos en antesis; frutos $5-7 \mathrm{~mm}$ de largo.

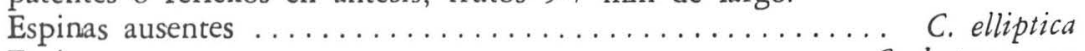

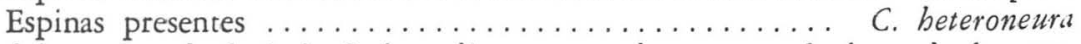
Glándulas del margen de la hoja 2, inmediatamente adyacentes a la base de la vena central, conspicuas, pateliformes o fungoides.......... (C. spinosa, C. retusa).

Se hizo un recuento cromosómico utilizando el método de aplastamiento de anteras con tinción de aceto-carmín; los botones se fijaron en el campo en la solución de Carnoy modificada ( 4 partes de cloroformo: 3 partes de alcohol etílico: 1 parte de ácido acético glacial). El recuento demuestra un número cromosómico de $n=16$ para Colubrina jobnstonii (Fig. 3).

El número cromosómico de $n=16$ representa el primer recuento reportado para Colubrina e indica un número básico inesperado en el género, basado en el patrón general de la familia. Raven (1975) postula un número básico de $x=12$ para Rhamnaceae. Los datos presentados por Fedorov (1969), Moore (1973, 1974, 1977) y Goldblatt (1981) apoyan la conclusión de Raven. Se ṛeportan en estos trabajos los 
BOLETIN DE LA SOCIEDAD BOTANICA DE MEXICO No. 44, 1983

CUADRO 1

CARACTERISTICAS DE COLUBRINA JOHNSTONII Y ESPECIES EMPARENTADAS

Carácter

longitud

de la lámi-

na $(\mathrm{cm})$

largo/ancho

de la lámi-

na

forma de la

lámina

glándulas

de la

lámina

estípulas

espinas

sépalos

erectos

persistentes
C.

C. jobnstonii

4-15

elliptica

C.

beteroneura

C.

spinosa

8-21

$2.5-9(-12) \quad 2.5-8.5$

$2.2-2.6$

(2.8-) $\quad 3-4 \quad(5.5) \quad 1.5-2.7(-3.3) \quad 1.5-2$

ovada u ova- obovado $\begin{array}{ll}\text { obovada u obo- } & \text { ovada u ova- } \\ \text { vado-lanceolada } & \text { do-elíptica }\end{array}$

obovada-oblonga a elíprico-oblanceolada

0-3 pares, 1 par, a usualmente $\quad 0.5-5 \mathrm{~mm}$ a $1-10 \mathrm{~mm}$ de la base, de la base, inconspicuas, inconspicuas, anulares a anulares a lineares lineares

caedizas

caedizas

caedizas

ausentes

presentes

presentes 0 ausentes

patentes 0 patentes 0 reflexos

reflexos

patentes 0 reflexos

longitud de pedicelo $(\mathrm{mm})$ en antesis

longitud de los frutos ( $\mathrm{mm}$ )

semillas: largo $(\mathrm{mm})$ 
recuentos para 61 especies en 15 géneros, de los cuales 48 especies (79\%) en 10 géneros muestran $n=12$ o un número poliploide (incluyendo triploide) basado en $x=12$. A nivel de género, los datos indican 10 géneros con $x=12,3$ con $x=11$, y 1 con $n=9$ (Maesopsis, el cual Johnston (1971) no incluye entre los géneros más emparentados con Colubrina), y un género cuyo número básico no es claro (Gouania, con $n=9$ y 23). No hay ningún reporte de $n=4,8$ ó 16 para la familia, aparte de los reportes de Escalante et al. (1971) de $n=4,6$ y 10 para tres especies de Condalia; estos recuentos no se incluyen en los datos presentados arriba porque DiFulvio (1977) ha demostrado que el número correcto en cada caso es $n=12$. Basado en esta información, el número de $n=16$ para Colubrina es extraordinario para la familia. Será necesario hacer recuentos para otras especies del género para determinar si $x=8$ ó 16 es característico del género, distinguiéndolo citológicamente de sus parientes.

El epíteto de la especie nueva honra al Dr. Marshall C. Johnston, estudioso perspicaz de Rhamanaceae y de la flora de México, quien sirvió valientemente como asesor de mis estudios de doctorado.

\section{AGRADECIMIENTOS}

Agradezco a Fernando Chiang C., Stephen D. Koch, Emily J. Lott y Mario Vázquez T., sus valiosos comentarios; a E. Mark Engleman su ayuda con la fotografía; a Eduardo Merino del Centro de Estudios del Desarrollo Rural del Colegio de Postgraduados la ilustración magnífica de la especie nueva; a Peter Goldblatt y Askell Love información adicional citológica acerca de Rhamnaceae; a Agustín Villalobos C. e Isidro Navarrete S. su valiosa ayuda en el campo; a M.C. Johnston sus comentarios preliminares acerca del problema; y a la Comisión del Papaloapan, S.A.R.H., su apoyo continuo para estos estudios del Centro de Botánica.

\section{BIBLIOGRAFIA}

DIFULVIO, T.E. 1977. El número cromosómico de Condalia (Rhamnaceae). Kurtziana 10: 249-250.

ESCALANTE, M.G., M. NAJERA, y H.L. GALDEANO. 1971. Las especies argentinas del género Condalio (Rhamnaceae). Revista Mus. La Plata, Secc. Bot. 11: 153-184.

FEDOROV, A.A. 1969. Chromosome Numbers of Flowering Plants. Academy of Sciences of the U.S.S.R., V.L. Komarov Botanical Institute (reimpresión 1974 Otto Koeltz, Koenigstein).

GOLDBLATT, P. 1981. Index to plant chromosome numbers 1975-1978. Monographs in Systematic Botany (Missouri Botanical Garden) 5.

JOHNSTON, M.C. 1971. Revision of Colubrina (Rhamnaceae). Brittonia 23: 2-53.

MARQUEZ RAMIREZ, W., A. GOMEZ-POMPA y M. VAZQUEZ TORRES. 1981. Estudios botánicos y ecológicos de la región del Río Uxpanapa, Veracruz. Núm. 10. La Vegetación y la Flora. Biotica 6: 181-217.

MIRANDA, F. y E. HERNANDEZ X. 1963. Los tipos de vegetación de México y su clasificación. Bol. Soc. Bot. Mexico 28: 29-178. 
BOLETIN DE LA SOCIEDAD BOTANICA DE MEXICO No. 44, 1983

MOORE, R.J. 1973. Index to plant chromosome numbers 1967-1971. Regnum Veg. 90.

MOORE, R.J. 1974. Index to plant chromosome numbers for 1972. Regnum Veg. 91

MOORE, R.J. 1977. Index to plant chromosome numbers for 19/3/74. Kegnum Veg. 96.

RAVEN, P. 1975. The bases of angiosperm phylogeny: cytology. Ann. Missouri Bot. Gard. 62: 724-764. 